\title{
Short-term changes in median nerve neural tension after a suboccipital muscle inhibition technique in subjects with cervical whiplash: a randomised controlled trial ${ }^{\text {is }}$
}

\author{
P.J. Antolinos-Campillo a , Á. Oliva-Pascual-Vaca ${ }^{\mathrm{b}}$, C. Rodríguez-Blanco $^{\mathrm{b}}$, \\ A.M. Heredia-Rizo ${ }^{\text {b,* }}$, G.V. Espí-López ${ }^{\text {c }}$, F. Ricard $^{\text {a }}$ \\ ${ }^{a}$ Madrid Osteopathic School, Madrid, Spain \\ ${ }^{\mathrm{b}}$ Department of Physiotherapy, Faculty of Nursing, Physiotherapy and Podiatry, University of Seville, Seville, Spain \\ ${ }^{\mathrm{c}}$ Department of Physiotherapy, Faculty of Physiotherapy, University of Valencia, Valencia, Spain
}

\begin{abstract}
Objectives To assess the immediate effect of a suboccipital muscle inhibition (SMI) technique on: (a) neck pain, (b) elbow extension range of motion during the upper limb neurodynamic test of the median nerve (ULNT-1), and (c) grip strength in subjects with cervical whiplash; and determine the relationships between key variables.

Design Randomised, single-blind, controlled clinical trial.

Setting Faculty of Nursing, Physiotherapy and Podiatry, University of Seville, Spain.

Participants Forty subjects $\{$ mean age 34 years [standard deviation (SD) 3.6] $\}$ with Grade I or II cervical whiplash and a positive response to the ULNT-1 were recruited and distributed into two study groups: intervention group (IG) $(n=20)$ and control group (CG) ( $n=20)$.

Interventions The IG underwent the SMI technique for 4 minutes and the CG received a sham (placebo) intervention. Measures were collected immediately after the intervention.

Main outcome measures The primary outcome was elbow range of motion during the ULNT-1, measured with a goniometer. The secondary outcomes were self-perceived neck pain (visual analogue scale) and free-pain grip strength, measured with a digital dynamometer.

Results The mean baseline elbow range of motion was $116.0^{\circ}$ (SD 10.2) for the CG and $130.1^{\circ}$ (SD 7.8) for the IG. The within-group comparison found a significant difference in elbow range of motion for the IG [mean difference $-15.4^{\circ}, 95 \%$ confidence interval (CI) -20.1 to $-10.6 ; P=0.01$ ], but not for the CG (mean difference $-4.9^{\circ}, 95 \% \mathrm{CI}-11.8$ to $2.0 ; P=0.15$ ). In the between-group comparison, the difference in elbow range of motion was significant (mean difference $-10.5^{\circ}, 95 \% \mathrm{CI}-18.6$ to $-2.3 ; P=0.013$ ), but the differences in grip strength $(P=0.06)$ and neck pain $(P=0.38)$ were not significant.

Conclusion The SMI technique has an immediate positive effect on elbow extension in the ULNT-1. No immediate effects on self-perceived cervical pain or grip strength were observed.
\end{abstract}

(C) 2013 Chartered Society of Physiotherapy. Published by Elsevier Ltd. All rights reserved.

Keywords: Whiplash injuries; Median nerve; Manipulation, Spinal; Pain

\section{Introduction}

Cervical whiplash injury is a common disorder, mainly defined by persistent neck and upper limb pain. Following

\footnotetext{
is Clinical Trial Registration Number: ACTRN 12611001238965.

* Corresponding author at: Department of Physiotherapy, Faculty of Nursing, Physiotherapy and Podiatry, University of Seville, C/ Avicena s/n, 41009 Seville, Spain. Tel.: +34 954486509; fax: +34 954486527.

E-mail address: amheredia@us.es (A.M. Heredia-Rizo).
}

cervical whiplash, neck pain can radiate to the spinal cord roots and has been linked with slight changes in median nerve function [1]. Cervical whiplash may also influence intervertebral discs, muscles, facet joints and ligaments, which may irritate surrounding nerve roots [2]. Therefore, neural responses and dysesthetic pain can appear in the absence of apparent nerve fibre or tissue damage [3].

Upper limb neurodynamic tests (ULNTs) are used to assess the functionality of the brachial plexus and to 
diagnose peripheral neuropathic pain [4]. A pathological response to the ULNT of the median nerve (ULNT-1) is based on reproduction of the patient's symptoms, along with the presence of resistance to movement and decreased elbow joint range of motion at pain onset or pain tolerance [4]. Hence, mechanosensitivity of the median nerve can be evaluated by detecting differences in elbow range of motion [5]. In addition, an abnormal response to the ULNT-1 has been linked to increased protective muscle activity in the cervical region and restrained joint movement to avoid overextensibility of neural tissue [6]. The involvement of sensitive cervical nerve tissues is present in $89 \%$ of subjects suffering persistent arm pain and paresthesia in chronic cervical whiplash [7]. Irritation of the brachial plexus, with constant diffuse pain and/or paraesthesia in the upper limb, was observed in $38 \%$ of subjects suffering from cervical whiplash between 1 and 12 weeks after the injury [8].

Lowered pain thresholds have been reported locally in the nerve trunks in the upper extremities, and distal to the injured area in subjects with chronic cervical-whiplash-associated disorders [9]. This finding suggests that local diffuse neck pain in cervical whiplash is related, in part, to sensitisation of the cervical nerve roots, but also to a central sensitisation process that may affect distal areas (e.g. wrist) where no tissue damage is required to provoke pain and alter functionality [9]. Grip strength has been included as a tool to measure functional capacity in cervical whiplash [10]. Entrapment of the median nerve in the carpal tunnel has been suggested as an associated component to the chronic pain of the upper limb in patients with cervical whiplash [11]. Furthermore, reduced movement of the median nerve has been reported proximal to the carpal tunnel in subjects with non-specific arm pain [12]. To the best of the authors' knowledge, this is the first study to report the effect of manual therapy on grip strength in patients with cervical whiplash.

Myofascial induction aims to relax muscular excitability, which may be linked to the perpetuation of central sensitisation in cervical whiplash [13]. It has also been shown to have a positive impact on joint range of motion [14], pain relief [15] and overall physical function [16]. This study hypothesised that a suboccipital muscle inhibition (SMI) procedure would reduce the muscle guarding in this region in patients with cervical whiplash. Therefore, the objective of this study was to assess the immediate effect of the SMI technique on the subject's response to the ULNT-1, self-perceived neck pain and grip strength, and determine the relationship between key variables.

\section{Materials and methods}

\section{Design and randomisation procedure}

A randomised [using a randomised number table designed by an Internet website (randomized.com)], single-blind (no relationship between the evaluator and the therapist in charge of the intervention, also see 'Blinding' section) controlled clinical trial was undertaken. An external consultant prevented access to the sequence for those participating in the study.

\section{Blinding}

Before randomisation, all participants were informed of the general aspects of the trial (possible benefits, risks, side effects of assessments and interventions, and that different types of treatments would be compared). Subjects and evaluators, who collected or analysed data, were unaware of the treatment allocation group.

\section{Sampling process}

The subjects were selected according to non-probabilistic convenience sampling techniques. The results of a previous pilot study [17] were analysed using the program Ene 2.0 (Universidad Autónoma de Barcelona, Spain). Taking into account a one-tailed hypothesis, a sample size of 17 subjects per group was necessary for a mean between-group difference in elbow range of motion of $5^{\circ}$, an $\alpha$ value of 0.05 and statistical power of $90 \%$.

\section{Inclusion and exclusion criteria}

The inclusion criteria for participants were: (a) age 18 to 55 years; (b) medical diagnosis of Grade I or II cervical whiplash according to the Québec Task Force [18]; and (c) positive response to the ULNT-1 for both evaluator and therapist. Patients with any of the following characteristics were excluded: (a) neck pain within 3 months preceding cervical whiplash; (b) medical diagnosis of at least Grade III cervical whiplash according to the Québec Task Force [18]; (c) malformations, previous surgery or injury in the cervical spine or the upper limbs that could prevent the subject from performing the ULNT-1; (d) history of neurological and/or rheumatic disorders; (e) soft tissue therapy within 3 months preceding the study; and (f) any contraindication to the intervention technique (e.g. tumoral disease, osteitis).

\section{Participants}

Fifty-six $(n=56)$ subjects with Grade I or II cervical whiplash were recruited for the study from one of the researcher's practices. After the allocation phase, the final sample included 40 subjects ( 17 women and 23 men) with a mean age of 34 years [standard deviation (SD) 3.6, range 19 to 55 years]. The participants were randomised into two study groups: intervention group (IG) $(n=20)$ and control group (CG) $(n=20)$. No loss to follow-up was recorded during the data collection or analysis phases (Fig. A, see online Supplementary material) [19]. The study protocol followed the CONSORT guidelines, was designed according to the institutional review board, and registered in the Australian and 
Box 1: Standard sequence of movements during median nerve upper limb neurodynamic test (ULNT-1)

- Shoulder girdle depression/stabilisation

- Shoulder girdle abduction (slightly $>90^{\circ}$ )

- Wrist/fingers extension $\left(90^{\circ}\right)$

- Maximal forearm supination

- Shoulder external rotation

- Elbow extension

- Structural differentiation (cervical sidebending)

New Zealand Clinical Trials Registry (Registration Number ACTRN 12611001238965).

\section{Measurement protocol}

After a consulting physician had diagnosed cervical whiplash, patients were selected if they qualified under the inclusion/exclusion criteria. A clinical neurological examination was performed on all subjects. The subject filled in an informed consent form, as established by the Declaration of Helsinki. The study was conducted in the same room at a constant temperature. Participants received the evaluation and intervention protocol together in one session. Both the therapist and the evaluator were senior physical therapists with over 6 years of experience in the field of manual therapy. The evaluator had been trained previously in management of the assessment tools. The assessment protocol was conducted in the following order.

\section{Self-perceived neck pain}

A visual analogue scale (VAS) was used. The subject remained seated on the treatment table with both feet on the ground, and was asked to mark the current intensity of pain on a horizontal $100-\mathrm{mm}$ line $(0 \mathrm{~mm}$ indicating no pain, $100 \mathrm{~mm}$ indicating the maximum possible pain). The VAS is a precise, effective, sensitive and reliable measurement tool for the evaluation of acute and chronic pain [20].

\section{Evaluation of elbow range of motion during the ULNT-1}

The participant was placed in the supine position with the upper limbs resting along the body, and the elbow of the tested side flexed $90^{\circ}$. The therapist asked the subject to 'Let me know when you start feeling pain or discomfort in the painful area'. The ULNT-1 was performed according to recognised methods (Box 1) [4]. The first step of the sequence was gentle shoulder girdle depression; this was standardised using an air-filled pressure sensor (Stabiliser, Chattanooga Group Inc., Chattanooga, TN, USA) placed between the evaluator's forearm and the upper surface of the subject's shoulder, and then inflated to a baseline of $40 \mathrm{mmHg}$ [6]. Shoulder girdle depression was continued until the pressure reached $60 \mathrm{mmHg}$, whereupon the sequence continued as shown in Box 1. When the subject reported pain or discomfort, the therapist stopped and measured the elbow extension range of motion. A universal goniometer was used, aligned along the mid humeral shaft, medial epicondyle and ulnar styloid. Intra-tester reliability of elbow extension with a goniometer is reported to be high [intra-class correlation coefficient 0.97 , 95\% confidence interval (CI) 0.95 to 0.99 ] [21], with a mean difference from a gold standard of $1.1^{\circ}$ (SD 5.1, 95\% CI 9.2 to -11.4 ) and a maximal error of $10.3^{\circ}$ [21].

The evaluator assessed which side had the more painful upper trapezius or the ULNT-1 provoked more severe symptoms or discomfort according to the subject's perception. The evaluation was only made on the more painful side. The ULNT-1 has been shown to have high intra- and interexaminer reliability (intra-class correlation coefficient $>0.95$ ) in patients with cervicobrachial pain [22] and in asymptomatic subjects [23]. It has been suggested that differences greater than $4.5^{\circ}$ represent a slight improvement when the ULNT-1 is repeated several times; when a single test is performed, differences should be greater than $7.5^{\circ}$ to show a relatively meaningful clinical change [22].

\section{Grip strength}

Pain-free grip strength was measured with a digital dynamometer (JAMAR 5030J1, Chicago, IL, USA) on the same side as that used to evaluate elbow range of motion. The subject was in a seated position, with the shoulder adducted and neutrally rotated, the elbow flexed to $90^{\circ}$, the forearm in the neutral position, and the wrist with a 'subtle' dorsal flexion, if needed, or in the neutral position [24]. The subject was instructed to 'Grasp it as strongly as you can without feeling pain or discomfort'. Three measurements were made, taking the mean as the reference value. Dynamometric evaluation of grip strength has proven to be valid [24].

\section{Suboccipital muscle inhibition technique in the intervention group}

The subject was in the supine position, and the therapist sat at the head of the table and placed both hands under the subject's head, contacting the space between the occipital condyles and the spinal process of the second cervical vertebra with the fingertips. Constant and painless pressure was exerted upward, towards the therapist [25]. A review of the literature indicated that 4 minutes was required to attain tissue relaxation at the suboccipital level $[17,26]$. Participants were asked to keep their eyes closed to avoid eye movements that might affect suboccipital muscle tone.

\section{Sham intervention in the control group}

The sham (placebo) intervention consisted of performing active movement of flexion/extension of the hip and knee 
Table 1

Baseline characteristics of participants.

\begin{tabular}{llll}
\hline & Control group $(n=20)$ & Intervention group $(n=20)$ & $P$-value \\
\hline $\begin{array}{l}\text { Age (years) } \\
\text { Gender }\end{array}$ & $36(5.3)$ & $(5.2)$ & 0.21 \\
$\quad$ Female, $n(\%)$ & $6(30)$ & $11(55)$ & 0.18 \\
$\quad$ Male, $n(\%)$ & $14(70)$ & $9(45)$ & \\
Affected upper limb (ULNT-1) & & $8(40)$ & 0.28 \\
$\quad$ Dominant side, $n(\%)$ & $12(60)$ & $12(60)$ & 0.84 \\
Non-dominant side, $n(\%)$ & $8(40)$ & $25.1(6.3)$ & 0.52 \\
Grip strength (kg) & $25.9(6.3)$ & $130(9.0)$ & 0.02 \\
Perceived neck pain (VAS) $(\mathrm{mm})$ & $56.2(10.6)$ & $130.8)$ \\
Elbow extension $\left({ }^{\circ}\right)$ & $116.0(10.2)$ & \\
\hline
\end{tabular}

Data are expressed as mean (standard deviation) or as $n(\%)$.

ULNT-1, upper limb neurodynamic test of the median nerve; VAS, visual analogue scale; $P$, statistical significance of the between-group difference.

joints on the opposite side to which the measurement was taken, according to the response to the ULNT-1. The intervention time was also set at 4 minutes.

\section{Statistical analysis}

PASW Advanced Statistics Version 18.0 (SPSS Inc., Chicago, IL, USA) was used for statistical analysis. Mean (SD), mean difference and 95\% CI of the difference were calculated for each variable. The Kolmogorov-Smirnov test showed a normal distribution of all quantitative variables $(P>0.05)$. Baseline characteristics in the study groups were compared using Student's $t$-test for quantitative variables and Chi-squared test for categorical variables.

Inferential analysis of variance for repeated measures (ANOVA test) for group (CG or IG) and time (pre- or post-intervention) allowed between-group differences to be observed. The effect size was evaluated using Cohen's test, and Pearson's correlation coefficients were used to evaluate the association between variables. $P<0.05$ was taken to indicate statistical significance.

\section{Results}

The study sample $(n=40)$ was evaluated to compare between-group differences in physical and clinical characteristics at baseline (Table 1). Significant differences were only found for elbow goniometry $(P=0.02)$.

Elbow range of motion and grip strength improved in both groups after the intervention, but neck pain was slightly worse after the intervention in both groups (Table 2). In the within-group comparison, only elbow range of motion was found to differ significantly for the IG $(P=0.01)$. When comparing between-group differences following the intervention (Table 2), a significant difference was found for elbow range of motion $\left[P=0.01 ; F(1,38)=6.81 ; R^{2}=0.15\right]$. However, no significant between-group differences were found for grip strength $(P=0.06)$ or neck pain and/or discomfort $(P=0.38)$.

The correlation study showed an association between neck pain and the other measured variables. A positive correlation was found between age and neck pain $(P<0.01 ; r=0.49)$, and a negative correlation was found between pain and grip strength $(P<0.01 ; r=-0.54)$, and pain and elbow range of motion $(P=0.01 ; r=-0.38)$.

Table 2

Change scores, and within- and between-group post-intervention comparisons for grip strength $(\mathrm{kg})$, neck pain $(\mathrm{mm})$ and elbow extension $\left(^{\circ}\right)$ during the upper limb neurodynamic test of the median nerve.

\begin{tabular}{|c|c|c|c|c|c|}
\hline & $\begin{array}{l}\text { Pre-intervention } \\
\text { Mean (SD) }\end{array}$ & $\begin{array}{l}\text { Post-intervention } \\
\text { Mean (SD) }\end{array}$ & $\begin{array}{l}\text { Within-group difference } \\
\text { Mean }(95 \% \mathrm{CI})\end{array}$ & $\begin{array}{l}\text { Between-group difference } \\
\text { Mean }(95 \% \mathrm{CI})\end{array}$ & $P$-value \\
\hline \multicolumn{6}{|l|}{ Grip strength } \\
\hline Control group & $25.9(6.3)$ & $28.0(7.3)$ & $-2.1(-3.6$ to -0.5$)$ & 1.67 & 0.06 \\
\hline Intervention & $25.1(6.3)$ & $25.5(7.9)$ & $-0.4(-1.4$ to 0.5$)$ & $(-0.1$ to 3.45$)$ & \\
\hline & & & & & \\
\hline \multicolumn{6}{|l|}{ Neck pain (VAS) } \\
\hline Control group & $56.2(10.6)$ & $55.2(10.5)$ & $1.0(-1.8$ to 3.8$)$ & -2.2 & 0.39 \\
\hline $\begin{array}{l}\text { Intervention } \\
\text { group }\end{array}$ & $52.0(9.0)$ & $48.7(10.8)$ & $3.2(-1.5$ to 8.0$)$ & $(-7.5$ to 3.0$)$ & \\
\hline \multicolumn{6}{|l|}{ Elbow extension } \\
\hline Control group & $116.0(10.2)$ & $120.9(14.8)$ & $-4.9(-11.8$ to 2.0$)$ & -10.5 & 0.01 \\
\hline $\begin{array}{l}\text { Intervention } \\
\text { group }\end{array}$ & $130.1(7.8)$ & $145.5(11.5)$ & $-15.4(-20.1$ to -10.6$)$ & $(-18.6$ to -2.3$)$ & \\
\hline
\end{tabular}

SD, standard deviation; CI, confidence interval; VAS, visual analogue scale; $P$-value, statistical significance of the between-group difference. 


\section{Discussion}

The SMI technique had a positive effect on elbow extension during the ULNT-1 in subjects with cervical whiplash. Elbow range of motion increased immediately after the intervention in the IG by $15.4^{\circ}$ (SD $10.2^{\circ}$ ). This result exceeds the threshold (4 to $7.5^{\circ}$ improvement in elbow mobility) that has been suggested to indicate a clinical change [22]. Nonetheless, SD estimates for elbow range of motion at pain onset during the ULNT-1 in symptomatic and asymptomatic subjects has been observed to range from $14^{\circ}$ to $20^{\circ}$ [4]. Furthermore, the potential maximal error for measurement of elbow extension with a universal goniometer is approximately $10^{\circ}$ [21], which means that the clinical relevance of the present results needs to be considered with caution. Although the goniometer is a simple, reliable and commonly used tool in clinical practice, the findings must be interpreted with caution when slight changes are found, due to the variability between subjects, the overlap in range of motion and the amount of measurement error [4]. Nevertheless, the aim of this study was not to compare the ULNT-1 range of motion between both upper limbs or with asymptomatic subjects, but to assess the changes between pre- and post-intervention.

The SMI technique has previously reported positive results distal to the area of treatment. Quintana Aparicio et al. [25] found an increase in hamstring flexibility after the SMI manoeuvre, and they considered that fascial continuity in the muscle and neural level would be a possible explanation for this phenomenon. The dura mater establishes a direct link with the musculoskeletal system through the rectus capitis posterior minor muscle in the suboccipital region, the so-called 'myodural bridge' [27]. A manual technique that may achieve a release of tension at this level would affect peripheral tension according to the tensegrity theory [28]. This theory proposes a structural concept of the body that explains how release of fascial tension in one part may involve the whole structure, being the main biomechanical basis to explain the results of myofascial techniques [28]. Furthermore, fascial continuity through neuromuscular chains has been hypothesised to elucidate a relationship between neck and upper limb muscles [29]. That would explain the release of distal tension and, consequently, the improvement of joint mobility after a local technique at the suboccipital level.

The myodural bridge also represents a potential explanation for the effect of manual therapy at the suboccipital region on craniocervical pain, as the increase of fatty infiltration in the rectus capitis posterior minor muscle of subjects with chronic neck pain after cervical whiplash suggests damage to the suboccipital musculature [27]. Nonetheless, no differences in VAS scores were observed in the IG. In fact, according to the results of correlations, an improvement in elbow mobility should have implied a decrease in cervical pain. On the one hand, local changes after myofascial induction in the cervicomandibular area have been observed regarding neck mobility [14], and neural and muscular mechanosensitivity $[15,26]$. On the other hand, no changes in pressure pain threshold at a local level were reported after fascial induction manoeuvres in the cervical region [14]. However, all these studies evaluated short-term effects in asymptomatic subjects, and they all concluded that the results were below the minimum detectable change to assume clinical significance. In a pilot study with subjects with subacute cervical-whiplash-associated disorders who underwent the Fascial Manipulation ${ }^{\odot}$ technique, Picelli et al. [30] observed a significant decrease in neck pain in a 2-week follow-up. Nevertheless, the Fascial Manipulation technique is different from the procedure used in the present trial, and was not performed solely within the cervical region, making comparison between the two studies difficult.

Vernon et al. [31] reported that irritability of the deep cervical paraspinal tissues, as happens after cervical whiplash, may cause hyperalgesia linked to a central sensitisation process. This may explain why the SMI technique was not sufficient to activate the descendent inhibitory system and relieve pain. Central sensitisation can be perpetuated by psychological aspects that increase pain at the central nervous system level [32]. Psychological and cognitive factors also play a role in cervical whiplash influencing pain perception, and may contribute as a perpetuating factor of cervical-whiplash-associated disorders [33]. In conclusion, concerning neck pain relief, there is limited evidence for most of the therapies used for cervical whiplash, although active interventions seem to have more effect than passive modalities [34].

Regarding grip strength, there were no between-group differences after the intervention. Immediate changes in painfree grip strength have been observed in the affected upper limb after end range high-velocity low-amplitude spinal manipulation, either in the cervical or thoracic region, in subjects with lateral epicondylalgia $[35,36]$. Spinal manipulation has been reported to produce hypoalgesic effects related to its influence on central mechanisms of processing and controlling pain [37]. However, this effect has not been found with the SMI technique.

\section{Study limitations}

Measurements of grip strength and elbow extension were only made in one upper limb. Therefore, it was not possible to compare the results between dominant and non-dominant sides. It was hypothesised that some of the participants may have been expecting financial compensation following a road traffic accident. Self-reported pain and symptoms during the ULNT-1 could have been influenced by this aspect, as being part of a compensation process has been associated with poorer outcomes [38]. Finally, all the effects were evaluated in the short term. It would be interesting to assess results over the medium to long term to observe results of higher clinical significance. 


\section{Conclusion}

The SMI technique immediately improves elbow extension during the ULNT-1 in subjects with cervical whiplash. However, no immediate effect on grip strength or neck pain was observed.

Ethical approval: Ethical Committee of Experimentation of the University of Seville, Spain.

Conflict of interest: None declared.

\section{Appendix A. Supplementary data}

Supplementary data associated with this article can be found, in the online version, at http://dx.doi.org/10.1016/ j.physio.2013.09.005.

\section{References}

[1] Greening J, Lynn B, Leary R. Sensory and autonomic function and ultrasound nerve imaging in RSI patients and keyboard workers. Pain $2003 ; 104: 275-81$

[2] Rydevik B, Szpalski M, Aebi M, Gunzburg R. Whiplash injuries and associated disorders: new insights into an old problem. Eur Spine J 2008;17:S359-416.

[3] Bove GM. Epi-perineurial anatomy, innervation, and axonal nociceptive mechanisms. J Bodyw Mov Ther 2008;12:185-90.

[4] Nee RJ, Jull GA, Vicenzino B, Coppieters MW. The validity of upperlimb neurodynamic tests for detecting peripheral neuropathic pain. J Orthop Sports Phys Ther 2012;42:413-24.

[5] Schmid AB, Brunner F, Luomajoki H, Held U, Bachmann LM, Künzer $\mathrm{S}$, et al. Reliability of clinical tests to evaluate nerve function and mechanosensitivity of the upper limb peripheral nervous system. BMC Musculoskelet Disord 2009;10:11.

[6] Balster S, Jull G. Upper trapezius activity during the brachial plexus tension test in asymptomatic subjects. Man Ther 1997;2: 144-9.

[7] Quintner J. A study of upper limb pain and paraesthesia following neck injury in motor vehicle accidents: assessment of brachial plexus tension test of Elvey. Br J Rheumatol 1989;28:528-33.

[8] Ide M, Ide J, Yamagam M, Takagik K. Symptoms and signs of irritation of the brachial plexus in whiplash injuries. J Bone Joint Surg 2001;83:226-9.

[9] Stone AM, Vicenzino B, Lim EC, Sterling M. Measures of central hyperexcitability in chronic whiplash associated disorder - a systematic review and meta-analysis. Man Ther 2013;18:111-7.

[10] Trippolini MA, Reneman MF, Jansen B, Dijkstra PU, Geertzen JH. Reliability and safety of functional capacity evaluation in patients with whiplash associated disorders. J Occup Rehabil 2013;23: 381-90.

[11] Alpar E, Onuoha G, Killampali V, Waters R. Management of chronic pain in whiplash injury. J Bone Joint Surg 2002;84:807-11.

[12] Greening J, Lynn B, Leary R, Warren L, O'Hinggins P, Hall-Craggs M. The use of ultrasound imaging to demonstrate reduced movement of the median nerve during wrist flexion in patients with non-specific arm pain. J Hand Surg Br 2001;26:401-6.

[13] Fernández-Pérez AM, Villaverde-Gutiérrez C, Mora-Sánchez A, Alonso-Blanco C, Sterling M, Fernández-de-Las-Peñas C. Muscle trigger points, pressure pain threshold, and cervical range of motion in patients with high level of disability related to acute whiplash injury. $\mathrm{J}$ Orthop Sports Phys Ther 2012;42:634-41.
[14] Saíz-Llamosas JR, Fernández-Pérez AM, Fajardo Rodríguez MF, Pilat A, Valenza Demet G, Fernández de Las Peñas C. Changes in neck mobility and pressure pain threshold levels following a cervical myofascial induction technique in pain-free healthy subjects. J Manip Physiol Ther 2009;32:352-7.

[15] Heredia-Rizo AM, Oliva-Pascual-Vaca A, Rodríguez-Blanco C, PiñaPozo F, Luque-Carrasco A, Herrera-Monge P. Immediate changes in masticatory mechanosensitivity, mouth opening, and head posture after myofascial techniques in pain-free healthy participants: a randomized controlled trial. J Manip Physiol Ther 2013;36: $310-8$.

[16] Castro-Sánchez AM, Matarán-Peñarrocha GA, Granero-Molina J, Aguilera-Manrique G, Quesada-Rubio JM, Moreno-Lorenzo C. Benefits of massage-myofascial release therapy on pain, anxiety, quality of sleep, depression, and quality of life in patients with fibromyalgia. Evid Based Complement Alternat Med 2011;2011: 561753 .

[17] Antolinos-Campillo PJ, Martínez-Franco AF, Heredia-Rizo AM. Effectiveness of the suboccipital muscle inhibition technique on the neurodynamic test of the median nerve in patients with whiplash: a pilot study. Eur J Ost Clin Rel Res 2012;7:22-8.

[18] Spitzer WO, Skovron ML, Salmi LR, Cassidy JD, Duranceau J, Suissa $\mathrm{S}$, et al. Scientific monograph of the Québec Task Force on Whiplash Associated Disorders: redefining whiplash and its management. Spine (Phila Pa 1976) 1995;20:1-73.

[19] Turner L, Shamseer L, Altman DG, Weeks L, Peters J, Kober T, et al. Consolidated standards of reporting trials (CONSORT) and the completeness of reporting of randomised controlled trials (RCTs) published in medical journals. Cochrane Database Syst Rev 2012;14: MR000030

[20] Carlsson AM. Assessment of chronic pain. I. Aspects of the reliability and validity of the visual analogue scale. Pain 1983;16:87-101.

[21] Chapleau J, Canet F, Petit Y, Laflamme GY, Rouleau DM. Validity of goniometric elbow measurements: comparative study with a radiographic method. Clin Orthop Relat Res 2011;469:3134-40.

[22] Coppieters M, Stappaerts K, Janssens K, Jull G. Reliability of detecting 'onset of pain' and 'submaximal pain' during neural provocation testing of the upper quadrant. Physiother Res Int 2002;7:146-56.

[23] Oliver GS, Rushton A. A study to explore the reliability and precision of intra and inter-rater measures of ULNT 1 on an asymptomatic population. Man Ther 2011;16:203-6.

[24] Irwin CB, Sesto ME. Reliability and validity of the multiaxis profile dynamometer with younger and older participants. J Hand Ther 2010;23:281-8.

[25] Quintana Aparicio E, Borrallo Quirante L, Rodríguez Blanco C, Alburquerque Sendín F. Immediate effects of the suboccipital muscle inhibition technique in subjects with short hamstring syndrome. J Manip Physiol Ther 2009;32:262-9.

[26] Heredia-Rizo AM, Oliva Pascual-Vaca A, Albornoz-Cabello M, Rodríguez-Blanco C, Piña-Pozo F, Luque-Carrasco A. Immediate effects of the suboccipital muscle inhibition technique in craniocervical posture and greater occipital nerve mechanosensitivity in subjects with a history of orthodontia use: a randomized trial. J Manip Physiol Ther 2012;35:446-53.

[27] Kahkeshani K, Ward PJ. Connection between the spinal dura mater and suboccipital musculature: evidence for the myodural bridge and a route for its dissection - a review. Clin Anat 2012;25: 415-22.

[28] Masi AT, Nair K, Evans T, Ghandour Y. Clinical, biomechanical, and physiological translational interpretations of human resting myofascial tone or tension. Int J Ther Massage Bodywork 2010;3:16-28.

[29] Busquet L. Las Cadenas Musculares. Tomo I. Tronco y Columna Cervical. Barcelona: Paidotribo; 2006

[30] Picelli A, Ledro G, Turrina A, Stecco C, Santilli V, Smania N. Effects of myofascial technique in patients with subacute whiplash associated disorders: a pilot study. Eur J Phys Rehabil Med 2011;47:561-8. 
[31] Vernon H, Sun K, Zhang Y, Yu XM, Sessle BJ. Central sensitization induced in trigeminal and upper cervical dorsal horn neurons by noxious stimulation of deep cervical paraspinal tissues in rats with minimal surgical trauma. J Manip Physiol Ther 2009;32:506-14.

[32] Sterling M. Whiplash-associated disorder: musculoskeletal pain and related clinical findings. J Man Manip Ther 2011;19:194-200.

[33] Carroll LJ, Liu Y, Holm LW, Cassidy JD, Côté P. Pain-related emotions in early stages of recovery in whiplash-associated disorders: their presence, intensity, and association with pain recovery. Psychosom Med 2011;73:708-15.

[34] Hurwitz EL, Carragee EJ, van der Velde G, Carroll LJ, Nordin M, Guzman J, et al. Treatment of neck pain: noninvasive interventions: results of the Bone and Joint Decade 2000-2010 Task Force on Neck Pain and Its Associated Disorders. Spine (Phila Pa 1976) 2008;33(Suppl.):S123-52.
[35] Fernández-Carnero J, Cleland JA, Arbizu RL. Examination of motor and hypoalgesic effects of cervical vs thoracic spine manipulation in patients with lateral epicondylalgia: a clinical trial. J Manip Physiol Ther 2011;34:432-40.

[36] Fernandez-Carnero J, Fernandez-de-Las-Peñas C, Cleland JA. Immediate hypoalgesic and motor effects after a single cervical spine manipulation in subjects with lateral epicondylalgia. J Manip Physiol Ther 2008;31:675-81

[37] Pickar JG. Neurophysiological effects of spinal manipulation. Spine J 2002;2:357-71.

[38] Casey PP, Feyer AM, Cameron ID. Identifying predictors of early non-recovery in a compensation setting: the Whiplash Outcome Study. Injury 2011;42:25-32.

Available online at www.sciencedirect.com

\section{ScienceDirect}

